\title{
Who is being tested by the English National Chlamydia Screening Programme? A comparison with national probability survey data
}

\author{
Johanna Riha, ${ }^{1}$ Catherine H Mercer, ${ }^{2}$ Kate Soldan, ${ }^{1}$ Clare E French, ${ }^{1}$ \\ Mary Macintosh ${ }^{1}$
}

- Additional tables are published online only. To view these files please visit the journal online (http://sti.bmj. com)

${ }^{1}$ National Chlamydia Screening Programme, Health Protection Agency, London, UK ${ }^{2}$ Centre for Sexual Health \& HIV Research, Department of Infection and Population Health, University College London, London, UK

\section{Correspondence to} Kate Soldan, Health Protection Services - Colindale, Health Protection Agency, 61 Colindale Avenue, London, NW9 5EQ, UK; kate.soldan@hpa.org.uk

Accepted 19 February 2011

Published Online First

22 March 2011

\section{UNLOCKED}

This paper is freely available online under the BMJ Journals unlocked scheme, see http://sti. bmi.com/site/about/unlocked. xhtml

\begin{abstract}
Objectives We compare data collected by England's National Chlamydia Screening Programme (NCSP) with national probability survey data to examine demographic and behavioural differences that may be important in understanding who the NCSP is reaching and interpreting chlamydia positivity.

Methods Data for 538119 men and women aged 16-24 years who were screened in 2008 and data collected from 2180 interviewees in Britain's second National Survey of Sexual Attitudes and Lifestyles 1999-2001 (Natsal-2), of whom 644 were tested for chlamydia, were compared using the $\chi^{2}$ statistic and logistic regression.
\end{abstract}

Results Compared with Natsal-2, the NCSP tested more women $(67 \%$ vs $49 \%)$. NCSP participants were more likely to be younger: $29 \%$ were $16-17$ years versus $16 \%$ of men and $15 \%$ of women in Natsal-2; from ethnic minority groups: $17 \%$ of men and $14 \%$ of women versus $8 \%$ and $6 \%$, respectively, in Natsal-2; not to have used condoms at last sex: $66 \%$ of men and $68 \%$ of women versus $48 \%$ and $63 \%$, respectively, in Natsal-2: and more likely to report two or more partners in the last year: $62 \%$ of men and $47 \%$ of women versus $47 \%$ and $30 \%$, respectively, in Natsal-2. In multivariate analyses, higher $\mathrm{AOR}$ of chlamydia positivity were found for those reporting non-use of condoms and for those reporting multiple partners in both the NCSP and Natsal-2.

Conclusions The NCSP is testing young people at increased risk of chlamydia. The impact of this testing bias on the effectiveness of the programme should be evaluated.

\section{INTRODUCTION}

Genital Chlamydia trachomatis infection is the most commonly diagnosed bacterial sexually transmitted infection in the UK. ${ }^{1}$ It is commonly thought, based on experience in genitourinary medicine (GUM) clinics, that around $50 \%$ of infections in men and $60-70 \%$ of infections in women are asymptomatic, although some data put this higher at around $90 \%$ in men. ${ }^{2}$ If left untreated genital chlamydial infections can have serious long-term consequences, including pelvic inflammatory disease, ectopic pregnancy and tubal factor infertility in women, and urethritis, epididymitis and Reiter's syndrome in men. ${ }^{3-5}$ The National Chlamydia Screening Programme (NCSP) was launched in England in 2003 to control chlamydial infection through early detection and treatment of predominantly asymptomatic infections to prevent onward transmission and the development of sequelae. $^{6}$ Unlike the cervical, breast or bowel screening programmes in England where medical registers are used to invite eligible subgroups of the population, the NCSP was not implemented as a call-recall register-based programme. Instead, the NCSP was set up as an opportunistic programme offering testing and treatment to men and women under the age of 25 years in healthcare and nonhealthcare based settings. The proportion of those testing positive through the programme has been higher than the prevalence observed in the general population $^{7} 8$ and is likely to be influenced by factors associated with who is offered and who accepts chlamydia testing.

Between 1999 and 2001, Britain's second National Survey of Sexual Attitudes and Lifestyles (Natsal-2), a population-based probability sample survey, was carried out. In addition to collecting data on sexual behaviour and attitudes, the survey collected urine samples from a subset of sexually active adults and estimated the population-based prevalence of genital chlamydia. At the time of writing, the Natsal-2 data are the most up-to-date and well-characterised population-based chlamydia prevalence data available for Britain. Therefore, these data provide an opportunity to explore differences between those who are tested through the NCSP and a nationally representative general population sample. The aims of this study are to (i) compare the demographic and behavioural profiles of those participating in the NCSP relative to a general population sample (Natsal-2); (ii) examine whether the same demographic and behavioural risk factors for chlamydia are seen in both the NCSP and the general population; and (iii) consider how this comparison informs interpretation of NCSP positivity rates.

\section{METHODS}

\section{Data sources}

NCSP

The NCSP tests sexually active young men and women aged less than 25 years for chlamydia outside of GUM clinics. Altogether, 542052 tests were recorded in the NCSP database for 2008. Reported data do not necessarily include a unique patient identifier. In total, 2450 test records matched other records by sex, date of birth, ethnicity and postcode of residence. These were considered highly likely to be repeat tests of the same individual and were excluded from our analysis. The first test was retained unless a subsequent test was positive in which case the first positive 
test was retained. An additional 1483 tests were excluded due to unknown sex. Data for 538119 men and women aged 16-24 years (an estimated $8.6 \%$ of the English population aged 16-24 years) who were tested for chlamydia through the programme in 2008 entered analysis. The programme collects sociodemographic data (age, gender both 100\% completeness and ethnicity with around $76 \%$ completeness) and sexual behavioural data (two or more sexual partners in the last year with around $67 \%$ completeness) for each test conducted. Information about condom use at last sex is additionally requested of NCSP participants in less than $20 \%$ of programme areas. Where asked, this question is completed with a 'yes' or 'no' response for around $50 \%$ of participants.

\section{Natsal-2}

Natsal-2 involved a stratified probability sample of 11161 male and female British residents aged 16-44 years interviewed between 1999 and 2001 based on addresses drawn using a multistage probability cluster design from the Postcode Address File. Because the prevalence of many HIV risk factors had been found to be higher in London relative to the rest of the country in the first Natsal survey conducted a decade earlier, addresses in Greater London were over sampled in Natsal-2. ${ }^{9}$ Information was collected by face-to-face computer-assisted personal interviews with the more sensitive questions asked through computer-assisted self-interviews (CASI). In addition, a random half of sexually active respondents aged 18-44 years were asked to provide a urine sample to test for $C$ trachomatis using ligase chain reaction. Natsal-2 achieved a response rate of $65 \%$ for participation in the survey which is in line with other major surveys conducted in Britain. ${ }^{10-12}$ The urine sample achieved a response rate of $71 \%$, which did not vary significantly by age group. ${ }^{13}$ Further details of the survey methodology and questionnaire are published elsewhere..$^{13-15}$ As described in other publications, ${ }^{14} 15$ the Natsal-2 questionnaire data were weighted for differential selection and response probabilities and the urine sample data were given an additional weight to take account of non-response in providing a urine sample calculated as the inverse probability of providing a urine sample. ${ }^{8}$ Details regarding the factors found to be associated with providing a urine sample are given in the paper by McCadden et al. ${ }^{13}$

\section{Data analyses}

We compared data collected by the NCSP throughout 2008 and Natsal-2. Two analyses were conducted: first, we compared the demographic and behavioural characteristics of those participating in the NCSP with those respondents aged 16-24 years who participated in the national probability survey with regards to five factors for which data were available from the two data sources: gender, age, ethnicity, condom use at last sex and having two or more sexual partners in the last year (expressed hereon for brevity as 'multiple partners'). Due to the large proportion of the NCSP participants for whom data on condom use was not available (see above), we compared the characteristics of NCSP participants with and without data for this variable to see whether there were any differences. We also compared the NCSP and Natsal-2 data limited to those participants with data for all five factors as the multivariate analyses (described below) also focused on these sub-samples. In the bivariate analyses, the $\chi^{2}$ statistic was used to determine whether any differences between the NCSP and the Natsal-2 data sources were statistically significant, which was considered as $\mathrm{p}<0.05$ for all analyses.

Second, we investigated how testing positive for chlamydia is associated with the five factors for each of the two data sources.
We used binary logistic regression to do this calculating the $\mathrm{OR}$ for testing positive for those in a particular category relative to the reference category-for example, among those aged 20-24 years relative to those aged $18-19$ years. To take account of any confounding, we also used multivariate binary logistic regression to enable us to calculate the OR adjusting for the other variables we had data for. The denominator for this second analysis was limited to those Natsal-2 participants aged 18-24 years who had met the sample selection criteria for providing a urine sample, ${ }^{8}$ who had agreed to do so and whose result had been tested for chlamydia $(n=644)$. Only those people for whom we had data on all five variables were included in this analysis. This corresponds to 627 of the eligible Natsal-2 participants (97\% of 644) and 17259 NCSP participants aged 18-24 years (4\%). We also ran this multivariate analysis excluding the condom use variable; thus, including those participants who had data available for gender, age, ethnicity and multiple partners (630 (98\%) of eligible Natsal-2 participants; 234034 (61\%) of NCSP participants aged $18-24$ years) to see if the results differed to the first multivariate model described.

To account for clustering, weighting and stratification of the Natsal-2 data, all analyses were performed in STATA 9.2 using the complex survey analysis functions. ${ }^{16}$ The NCSP data were considered as a simple sample with no weighting (ie, a weight of one).

\section{RESULTS}

\section{Demographic and behavioural characteristics}

Compared with Natsal-2, the NCSP tested larger proportions of women $(67.2 \%$, 95\% CI $66.8 \%$ to $67.2 \%$ vs $49.3 \%$, 95\% CI $46.5 \%$ to $52.1 \%$ ) (data not shown in tables). Table 1 compares the demographic and behavioural characteristics of NCSP participants relative to the general population aged 16-24 years, according to Natsal-2, by gender. Relative to Natsal-2 respondents, men and women participating in the NCSP were more likely to be younger, specifically aged $16-17$ years: $29 \%$ of men and women in the NCSP versus $16 \%$ of men and $15 \%$ of women in Natsal-2. NCSP participants were also more likely to be from ethnic minority groups: $17 \%$ of men and $14 \%$ of women versus $8 \%$ and $6 \%$, respectively, in Natsal-2, and particularly from black ethnic groups: $8 \%$ of men and $7 \%$ of women in contrast to $2 \%$ and $1 \%$, respectively, among Natsal-2 participants. NCSP participants were also more likely to report not having used condoms at last sex than their counterparts in the general population: $66 \%$ of men and $68 \%$ of women versus $48 \%$ and $63 \%$, respectively in Natsal-2. NCSP participants were also more likely to report multiple partners in the last year: $62 \%$ of men and $47 \%$ of women versus $47 \%$ and $30 \%$, respectively, in Natsal-2.

Due to the large proportion of NCSP participants for whom data on condom use were not available (see above), we compared the characteristics of NCSP participants with and without data for this variable to see whether there were any differences. While statistically significant differences do exist, reflecting the size of these two groups, the magnitude of these differences are slim, typically within four percentage points (see online table 1). One notable exception though is that the proportion of women from ethnic minority groups is larger among those for whom data on condom use were not collected: $15 \%$ in contrast to $5 \%$ of women for whom these data were collected. Given these differences, we also compared the NCSP and Natsal-2 participants limited to those with data for all four factors (see online table 2). We found that the differences between the 
Table 1 A comparison of the demographic and behavioural characteristics of National Chlamydia Screening Programme (NCSP) participants relative to the general population aged $16-24$ years, according to Natsal-2, by gender

\begin{tabular}{|c|c|c|c|c|c|c|}
\hline Gender, data source, all & $\begin{array}{l}\text { Natsal-2 (2000) } \\
1289,995^{*} \\
\text { n }(\%) \dagger\end{array}$ & $\begin{array}{l}\text { Men NCSP } \\
(2008) 176430 \\
\text { n (\%) }\end{array}$ & p Value & $\begin{array}{l}\text { Natsal-2 (2000) } \\
1251,1185^{*} \\
\text { n (column \%)† }\end{array}$ & $\begin{array}{l}\text { Women NCSP } \\
\text { (2008) } 361689 \\
\text { n (column \%) }\end{array}$ & p Value \\
\hline \multicolumn{7}{|l|}{ Age, y } \\
\hline $16-17$ & $207(16)$ & $51453(29)$ & $\mathrm{p}<0.001$ & $193(15)$ & $103568(29)$ & $\mathrm{p}<0.001$ \\
\hline $18-19$ & $267(21)$ & $51600(29)$ & & $276(22)$ & $96126(27)$ & \\
\hline $20-24$ & $815(63)$ & $73377(42)$ & & $782(63)$ & $161995(45)$ & \\
\hline \multicolumn{7}{|l|}{ Ethnicity } \\
\hline White & $1182(92)$ & $103303(83)$ & $\mathrm{p}<0.001$ & $1180(94)$ & $245053(86)$ & $\mathrm{p}<0.001$ \\
\hline Black & $27(2)$ & 9397 (8) & & $9(1)$ & $19348(7)$ & \\
\hline Other minority & $76(6)$ & 11112 (9) & & $60(5)$ & $20680(7)$ & \\
\hline \multicolumn{7}{|l|}{ Condoms used at last sex $\neq$} \\
\hline Yes & $661(52)$ & $2744(34)$ & $\mathrm{p}<0.001$ & $456(37)$ & $6051(32)$ & $p=0.007$ \\
\hline No & $602(48)$ & $5347(66)$ & & $782(63)$ & $12639(68)$ & \\
\hline \multicolumn{7}{|c|}{$2+$ sexual partners, last year $\neq$} \\
\hline$<2$ & $655(53)$ & $40234(38)$ & $\mathrm{p}<0.001$ & $845(70)$ & $137253(53)$ & $\mathrm{p}<0.001$ \\
\hline $2+$ & $586(47)$ & $64721(62)$ & & $363(30)$ & 120303 (47) & \\
\hline
\end{tabular}

*Weighted, unweighted denominators in Natsal-2.

$\dagger$ Natsal-2 counts and percentages are weighted.

¥Condom use at last sex is asked about in less than $20 \%$ of NCSP areas, in which it is completed by around $50 \%$ of participants. $2+$ sexual partners is asked of all NCSP participants, with around $67 \%$ completeness.

two data sources shown in table 1 persisted, although there were smaller differences in the proportions of participants of non-white ethnicity.

\section{Factors associated with chlamydia positivity}

Overall, both men and women (aged 18-24 years) who tested in the NCSP had higher chlamydia positivity than those tested in the general population sample: among men: $9.4 \%$ (95\% CI $9.2 \%$ to $9.5 \%$ ) versus $2.9 \%$ (95\% CI $1.3 \%$ to $5.2 \%$ ) and among women: $8.5 \%$ (95\% CI $8.4 \%$ to $8.6 \%$ ) versus $3.1 \%$ (95\% CI $1.6 \%$ to $5.7 \%$ ). The corresponding numbers of positive chlamydia cases were 11454 men and 21316 women in the NCSP; 8 men and 16 women in the Natsal-2 sample.

Table 2 shows the factors associated with increased risk of chlamydia infection in each data source for men and women separately. Age was only significantly associated with having chlamydia among women in the NCSP with lower odds for those aged 20-24 years relative to those aged 18-19 years (AOR $0.84,95 \%$ CI 0.74 to 0.96 ). Ethnicity appeared significantly associated for women in the NCSP (only) with lower odds for black women relative to white women (AOR 0.34, 95\% CI 0.15 to 0.76$)$. However, NCSP women of black ethnicity who responded to the question regarding condom use (and were therefore included in our analysis) had a lower prevalence of chlamydia infection than all NCSP women of black ethnicity ( $3 \%$ vs $9.5 \%$ ), which will have introduced bias into our analysis with respect to ethnicity. When the multivariate model was run for those participants with data for the other factors, excluding condom use (online tables $3 \mathrm{~A}$ and $3 \mathrm{~B}$ ), the $\mathrm{AOR}$ for these women increased to 1.19 (95\% CI 1.11 to 1.27$)$ and a similar relationship was observed for men in the NCSP of black ethnicity: AOR 1.42 (95\% CI 1.30 to 1.54 ).

In terms of sexual risk behaviours, for both genders in both data sources the AOR was greater than one for participants who reported not using condoms at last sex: among men, AORs of 1.53 for NCSP participants and 10.3 for men in Natsal-2; among women, AORs of 1.23 and 1.31, respectively. Similarly, NCSP participants who reported multiple partners in the year prior to testing for chlamydia were significantly more likely to test positive: AORs of 1.62 and 1.81 for men and women, respectively. This relationship was also observed among Natsal-2 women but was not statistically significant (AOR 2.85, 95\% CI 0.88 to 9.22). Among men in Natsal-2 sample, none of those reporting at most one partner in this time-frame tested positive for chlamydia, while prevalence was $6.7 \%$ (95\% CI $3.0 \%$ to $14.1 \%$ ) among those reported multiple partners.

\section{DISCUSSION}

This study has shown that the NCSP is testing individuals at higher risk of chlamydia than those in the general population. Similar behavioural risk factors for testing positive for chlamydia were identified for both populations. In line with previous published figures, ${ }^{17} 18$ individuals reporting not having used a condom at last sex and those having multiple partners had higher odds of testing positive for chlamydia.

\section{Strengths and weaknesses of the study}

This study included a large sample size, particularly for NCSP records, with over half a million records used in these analyses. The use of nationally representative data allowed us to investigate the disparities that exist between those testing through the NCSP in contrast to a random sample of the general population.

There are several limitations with this study. First, the two data sources used different methods to collect genital samples and detect $C$ trachomatis. Natsal- 2 used the most sensitive and specific nucleic acid amplification assay (NAAT) available at the time of that study-the ligase chain reaction on participantcollected urines posted to the laboratory. ${ }^{8}$ NCSP testing in 2008 was by more recently developed NAAT assays conducted on a mixture of patient-collected and clinician-collected samples. Natsal samples may have suffered more loss of sensitivity as a result of greater specimen transport delays. ${ }^{8}$ This may have increased the difference in observed prevalence of chlamydia in the two populations but should not affect the differences in behaviours or risk factors associated with the infection in each of the two populations.

The response rate for Natsal-2 was consistent with other major social surveys, ${ }^{10-12}$ and the data were weighted to be broadly representative of the British general population with respect to the age, gender and regional distribution ${ }^{14} 15$ and to 


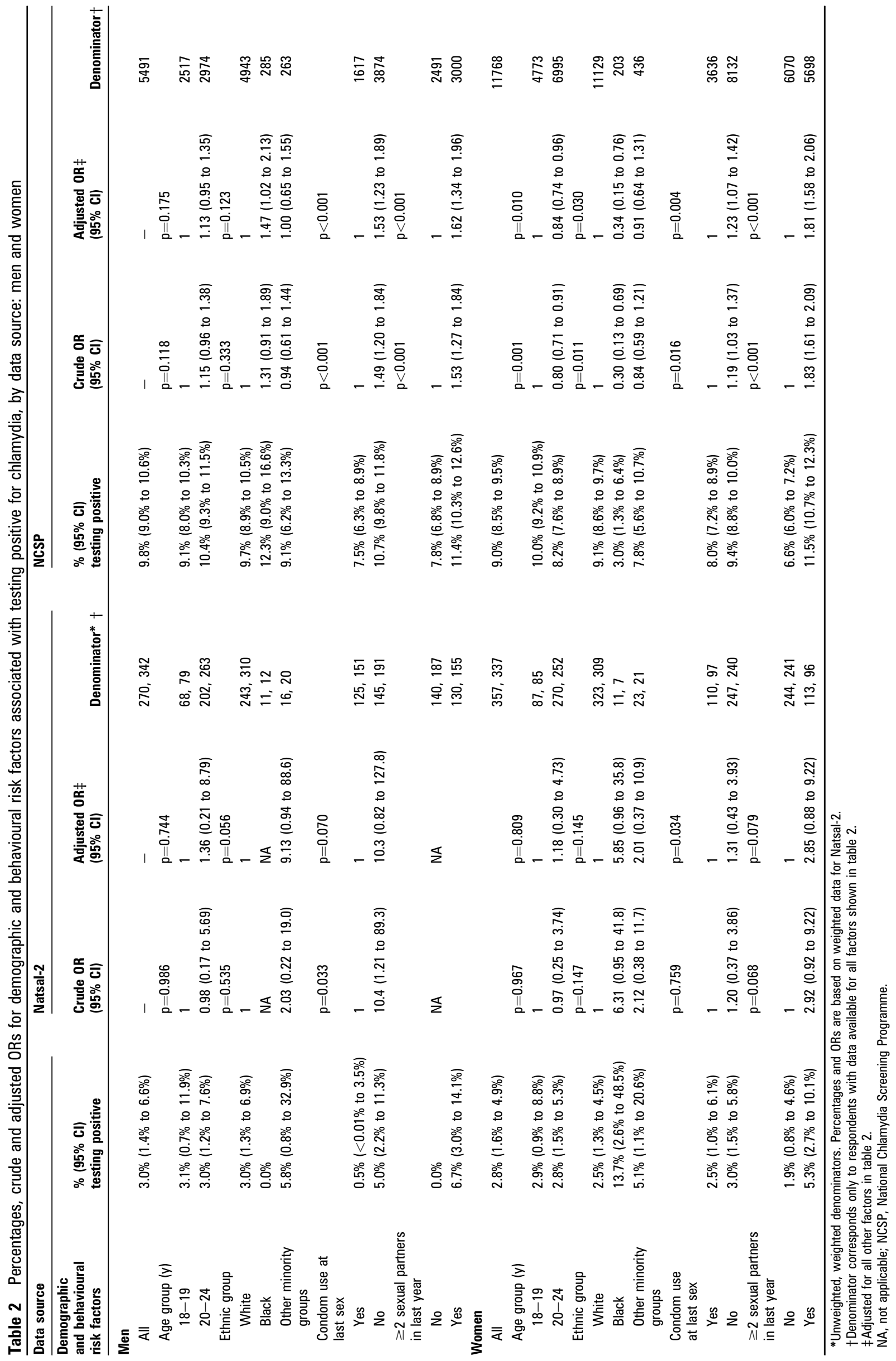


account for non-response to the urine sample collection. ${ }^{13}$ Nevertheless, response bias cannot be completely eliminated and remains a limitation of these data. The possibility of response bias also applies to the NCSP data since these data were also not $100 \%$ complete. Our inclusion of data on condom use at last sex appears to have introduced such a response bias in our analysis by ethnic group rather than to have improved the adjustment for confounding that we hoped it would. Our analysis of risk without this variable, and associated response bias, finds an elevated risk for individuals with black ethnicity as reported by a previous NCSP study. ${ }^{7}$

We had limited power due to the relatively small Natsal-2 sample size for the age group of interest, as noted above. There are also differences in terms of how the behavioural data were collected as Natsal-2 used CASI while the NCSP used a selfadministered or clinician completed pen-and-paper questionnaire. However, it is worth noting that in a randomised comparison of administering the Natsal-2 questionnaire as a CASI versus pen-and-paper self-completion, no consistent evidence of an increased willingness to report sensitive behaviours was observed. ${ }^{19}$

It would have been interesting to examine whether the strength of the associations found in both populations differed in the NCSP relative to the general population. For example, it is possible that the relationship between a single, binary risk behaviour variable on the probability of infection is less discriminating in the NCSP than in the general population. Unfortunately there were too few chlamydia cases in the Natsal2 (8 among men, 16 among women) and, thus, insufficient statistical power to test this hypothesis.

Finally, data for the two populations being compared were also collected during two different time periods with the general population sample collected around 8 years before the NCSP sample. However, at the time of writing, Natsal-2 data are the most up-to-date data available on chlamydia prevalence in the general population. As has been previously reported, ${ }^{14}$ it is possible that sexual behaviour in the British population has changed since the interviews for Natsal-2, which may account, at least in part, for the differences detected between those tested through the screening programme and Natsal-2. If the young British population has become more sexually active since Natsal2 was conducted it is possible that the results from this study, which describe the NCSP population as being more at risk, reflect this change. A comparison of NCSP participants and participants in Britain's third National Survey of Sexual Attitudes and Lifestyles (Natsal-3), being conducted in 2010-2012, should further our understanding of who is being tested by England's chlamydia screening programme.

\section{Comparison with other studies}

Others have compared certain characteristics of young people undergoing screening to those of young people more generally. For example, Sheringham et al have analysed residence-based Index of Multiple Deprivation scores (a variable we did not investigate) for young people screened through the NCSP in comparison to total population data and found that in 2008 the NCSP tested a larger proportion of individuals who resided in deprived areas compared to those in more affluent areas. ${ }^{20} \mathrm{We}$ are not aware of any studies prior to ours that have compared behavioural data of young people assessing opportunistic chlamydia screening in England with a representative sample of the general population. This is probably because there are no population-based behavioural data with chlamydia status that are comparable to Natsal for this purpose.

\section{Key messages}

Condom non-use and having multiple sexual partners is strongly associated with testing positive for chlamydia among both National Chlamydia Screening Programme (NCSP) participants and the general population.

- The NCSP is testing individuals at higher risk of chlamydia than those in the general population.

- As chlamydia screening becomes more widespread, further study of who is being tested would inform evaluation of screening effectiveness.

\section{Interpretation of the findings}

Our findings suggest that the testing venues delivering opportunistic NCSP chlamydia screening, and/or testing offers, have been preferentially accessed, and/or accepted, by sexually active young adults at higher than average risk of infection. The reasons for this, whether selection by attendance at screening venues, by health professionals offering of tests or by acceptance of test offers, or a combination of these potential selection pressures, are a matter for separate research.

\section{Implications of the findings}

Models used to explore the effectiveness of chlamydia screening in England have assumed that those accepting testing are a random sample of the population eligible for chlamydia testing. ${ }^{21}$ However, the results from this study suggest that those participating in the screening programme are at higher risk than the general population. The consequent higher yield of infections detected (and treated) by screening is an important feature that should be factored in when evaluating the impact of chlamydia screening in England. This could be done by incorporating test-positivity and population prevalence into models as two separate variables. Assuming all other model assumptions were correct, this bias to higher risk participants in screening that we have observed in England suggests that the effectiveness of chlamydia screening in England might have been higher than 'random sample' models would have predicted for the observed levels of coverage. Further analysis of this kind should be conducted as opportunities arise to monitor who is accessing screening and to inform modelling studies.

Funding This work was undertaken by the Health Protection Agency who received funding from the Department of Health; the views expressed in this publication are those of the authors and not necessarily those of the Department of Health (England).

Competing interests JR, KS, CF and MM are employed by the Health Protection Agency and work for the National Chlamydia Screening Programme.

Contributors JR and KS initiated the study. All authors contributed to determining the research questions and study design. JR and CM conducted the statistical analyses JR wrote the first draft of the manuscript. All authors provided substantive contributions to the final version of the manuscript.

Provenance and peer review Not commissioned; externally peer reviewed.

\section{REFERENCES}

1. Health Protection Agency. Testing Times: HIV and Other Sexually Transmitted Infections in the United Kingdom. London: Health Protection Agency, 2007.

2. McKay L, Clery H, Carrick-Anderson K, et al. Genital Chlamydia trachomatis infection in a subgroup of young men in the UK. Lancet 2003;361:1792.

3. Westrom $\mathbf{L}$, Joesoef $R$, Reynolds $\mathrm{G}$, et al. Pelvic inflammatory disease and fertility. Sex Transm Dis 1992;19:185-92.

4. Hillis $\mathbf{S}, 0$ wens $L$, Marchbanks $P$, et al. Recurrent chlamydial infections increase the risks of hospitalization for ectopic pregnancy and pelvic inflammatory disease. Am J Obstet Gynecol 1997;176:103-7. 
5. Tay JI, Moore J, Walker JJ. Regular review: ectopic pregnancy. BMJ 2000;320:916-19.

6. Department of Health. The National Strategy for Sexual Health and HIV. London: Department of Health, 2001.

7. Simms I, Talebi A, Riha J, et al. The English National Chlamydia Screening Programme: variations in positivity in 2007/2008. Sex Transm Dis 2009;36:522-7.

8. Fenton KA, Korovessis C, Johnson AM, et al. Sexual behaviour in Britain: reported sexually transmitted infections and prevalent genital Chlamydia trachomatis infection. Lancet 2001;358:1851-4.

9. Johnson AM, Wadsworth J, Wellings K, et al. Sexual Attitudes and Lifestyles. Oxford: Blackwell Scientific Press, 1994

10. Department of Work and Pensions. Family Resources Survey 2000-01, 2006 http://research.dwp.gov.uk/asd/frs/2000 01/methodology/response.asp laccessed 15 Jun 2010)

11. Lynn P, Clarke P. Separating refusal bias and non-contact bias: evidence from UK national surveys. J Roy Stats Soc Series D 2002:51:319-33.

12. Walker A, O'Brien M, Traynor J, et al. National Statistics. Living in Britain. Results from the 2001 General Household Survey. London: The Stationery Office, http://www.statistics.gov.uk/lib2001/resources/fileAttachments/GHS2001.pdf (accessed 15 Jun 2010).

13. McCadden A, Fenton KA, McManus S, et al. Chlamydia Trachomatis testing in the second British National Survey of Sexual Attitudes and Lifestyles: Respondent uptake and treatment outcomes. Sex Transm Dis 2005;32:387-94.
14. Johnson AM, Mercer $\mathrm{CH}$, Erens $\mathrm{B}$, et al Sexual behaviour in Britain: partnerships, practices, and HIV risk behaviours. Lancet 2001;358:1835-42

15. Erens B, McManus S, Field J, et al. National Survey of Sexual Attitudes and Lifestyles II: Technical Report. London: National Centre for Social Research, 2001.

16. Stata Corporation Stata Statistical Software: Release 9. College Station Texas, USA, 2006.

17. Hook EW III, Reichart CA, Upchurch DM, et al. Comparative behavioral epidemiology of gonococcal and chlamydial infections among patients attending a Baltimore Maryland, sexually transmitted disease clinic. Am J Epidemiol 1992; 136:662-72

18. Van Duynhoven YT, van de Laar MJ, Schop WA, et al. Different demographic and sexual correlates for chlamydial infection and gonorrhoea in Rotterdam. Int J Epidemiol 1997;26:1373-85.

19. Johnson AM, Copas AJ, Erens B, et al. Effect of computer-assisted self interviews on reporting of sexual HIV risk behaviours in a general population sample: a methodological experiment. AIDS 2001;15:111-15.

20. Sheringham J, Simms I, Riha J, et al. Socioeconomic variations in chlamydia screening coverage and positivity in England: Analysis of the National Chlamydia Screening Programme in 2008. Poster Presented at 18th ISSTDR in Conjunction with BASHH Conference 2009. London, UK.

21. Turner KM, Adams EJ, LaMontagne DS, et al. Modelling the effectiveness of chlamydia screening in England. Sex Transm Infect 2006:82:496-502.

\section{Fourth generation point of care testing for HIV: validation in an HIV-positive population}

Identification of primary HIV infection is valuable to reduce onward transmission and consider early antiretroviral therapy (ART). Fourth generation (4G) HIV tests detect p24 antigen in addition to antibody and therefore allow detection of HIV before antibody development. 4G point of care tests (POCTs) have been widely adopted despite an absence of prospective evidence and reports of reduced antigen sensitivity in primary HIV infection. ${ }^{1}$ The performance of the p24 component has not been evaluated in chronic infection and it is not known whether detection is influenced by factors such as illness or vaccination. Further evaluation of the p24 aspect of the 4G POCT is therefore required to allow accurate interpretation of test findings.

The Determine HIV-1/2 Ag/Ab Combo POCT (Alere Medical, Stockport, UK) was tested on whole blood samples obtained by finger prick from patients attending for routine HIV care at St Thomas' Hospital, London. Patients presenting with early p24 antigen-only infection had been diagnosed using the ARCHITECT HIV Ag/Ab Combo (Abbott Diagnostics, Germany) 4G serological assay, VIDAS p24 antigen test (Biomer- ieux, France) and Immunocomb II Bispot HIV 1 and 2 antibody test (Orgenics, Israel).

One hundred and forty seven HIV-infected patients were recruited prospectively between June and October 2010; 19/147 $(13 \%)$ POCTs were excluded because the visual control line on the test strip was absent. Of the remaining 128 individuals, 94 $(73 \%)$ were men, mean age 41 years (range, 24-73), mean CD4 count 515 cells/ $\mu$ (46-1186), mean time since diagnosis 67 months (0-326); 90 (70\%) were on ART, 5 $(3.9 \%)$ had primary HIV infection and 42 (33\%) had concomitant illness (30 patients), vaccination ( 9 patients) or sexually transmitted infection (STI, 17 patients).

Five out of $128(3.9 \%)$ tested positive for p24 antigen by 4G POCT. Of these, 3/5 reported recent influenza-like symptoms and had been diagnosed with primary HIV infection in the previous 2 months (p24 antigen positive and HIV antibody negative). The two remaining patients had no history of concomitant illness, vaccination or STI had been diagnosed HIV positive more than 5 years previously and were taking ART with a viral load of $<20$ copies $/ \mathrm{ml}$.

\section{CONCLUSION}

In a real life setting, the p24 antigen component of the Determine HIV-1/2 Ag/Ab Combo POCT has an acceptably low positive rate in chronic infection and is not affected by concomitant illness, vaccination or STI. The detection of p24 is therefore suggestive of early HIV infection and should guide clinicians accordingly. The high rate of void tests is of concern and needs further investigation.

\section{E Naylor, ${ }^{1}$ D Axten, ${ }^{2}$ F Makia, ${ }^{2}$ C Tong, ${ }^{3}$ J White, ${ }^{2}$ J Fox}

'Guy's and St Thomas' NHS Foundation Trust, London, UK; ' ${ }^{2}$ Department of GUM/HIV, Guy's and St Thomas' NHS Foundation Trust, London, UK; ${ }^{3}$ Department of Virology, Guy's and St Thomas' NHS Foundation Trust, London, UK

Correspondence to J Fox, Department of GUM/HIV Guy's and St Thomas' NHS Foundation Trust, Harrison Wing, 2nd Floor Lambeth Wing, St Thomas' Hospital, London, UK; julie.fox@kcl.ac.uk

\section{Competing interests None.}

Provenance and peer review Not commissioned; internally peer reviewed.

Accepted 26 March 2011

Sex Transm Infect 2011;87:311.

doi:10.1136/sextrans-2011-050056

\section{REFERENCE}

1. Fox J, Dunn H, O'Shea S. Low rates of p24 antigen detection using a fourth generation point of care HIV test. Sex Transm Infect 2011;87:178-9. 